\title{
Polymer dielectrics bring the voltage down
}

Adv. Electron. Mater. https://doi.org/10.1002/aelm.201800340 (2018)

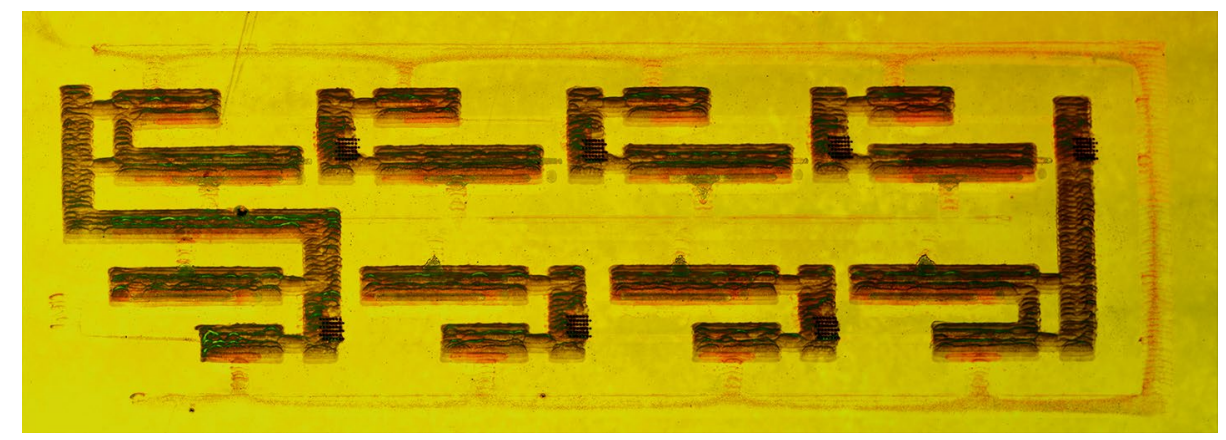

Credit: Wiley

Using polymer-based materials to build electronic circuits offers several advantages over using inorganic-based counterparts, particularly in high-volume and flexible electronics, where outright performance can be sacrificed in exchange for low-cost, additive manufacturing techniques such as inkjet printing. However, to fully realize these benefits on a commercial scale it may be necessary for all components - substrates, dielectrics, semiconductors and electrodes - to be fabricated using polymers. At present, a lack of high-performance dielectrics, which enable low-voltage transistor operation, remains a key bottleneck.

Mario Caironi and colleagues at Istituto Italiano di Tecnologia, Politecnico di Milano and Gyeongsang National University now show that a bilayer polymer dielectric (comprised of PMMA and parylene-C) can be used to fabricate all-polymer integrated circuits. Crucially, the surface properties of the bilayer allow thin, conformal layers, with comparatively high geometric capacitance values of $20 \mathrm{nF} \mathrm{cm}^{-2}$, to be deposited on top of semiconductors. Using only additive manufacturing methods, the researchers are able to fabricate all-polymer-based complementary metal-oxide-semiconductor (CMOS)-like inverters and ring oscillators, which operate at voltages as low as $2 \mathrm{~V}$ on flexible substrates. Additionally, they fabricate D flip-flops, further highlighting the potential suitability of all-polymer-based electronics for commercial applications.

\section{Stuart Thomas}

Published online: 12 October 2018 https://doi.org/10.1038/s41928-018-0157-2 